\title{
USO DE PACLOBUTRAZOL COMO ESTRATÉGIA PARA REDUÇ̃̃o DO PORTE E DA BROTAÇÃO LATERAL DE PLANTAS DE TOMAŢEIRO ${ }^{1}$
}

\author{
The use of paclobutrazol as a strategy for controlling height and side shooting of tomato plants ${ }^{1}$
}

\author{
Katiane Santiago Silva², Max José de Araujo Faria Junior ${ }^{3}$
}

\begin{abstract}
RESUMO
Objetivou-se, no trabalho, avaliar o efeito da aplicação de diferentes concentrações $\left(0 ; 50 ; 100\right.$ e $\left.150 \mathrm{mg} \mathrm{L}^{-1}\right)$ de paclobutrazol (PBZ) sobre o crescimento, a emissão de brotos laterais e a produtividade de dois híbridos de tomateiro (Jennifer e AF 7631), em campo aberto e em ambiente protegido. O trabalho foi desenvolvido de abril a outubro de 2007, na UNESP - Campus de Ilha Solteira, estado de São Paulo. O PBZ foi aplicado às mudas, aos 15 dias após a semeadura, avaliando-se seu efeito, nas concentrações testadas, em plantas de tomateiro após o seu transplantio. Adotou-se o delineamento em blocos casualizados, em esquema fatorial, com análise para grupo de experimentos, modelo fixo, com 4 blocos por ambiente e 9 plantas por parcela. Como resultado, o híbrido Jennifer apresentou maiores taxas de crescimento absoluto, resultando em plantas com maior altura que o AF 7631, aos 60 dias após o transplantio, sem que houvesse diferenças entre eles, no que se refere à brotação lateral e produtividade. Em ambiente protegido, foram obtidas plantas mais vigorosas, com maior altura, brotação lateral e maior produtividade que em campo aberto. O uso de concentrações crescentes de paclobutrazol reduziu a taxa de crescimento e a altura de plantas, bem como reduziu a brotação lateral e a produtividade da cultura.
\end{abstract}

Termos para indexação: Solanum lycopersicum L., regulador de crescimento, ambiente protegido.

\section{ABSTRACT}

The objective of this study was to evaluate the effect of different concentrations $\left(0 ; 50 ; 100\right.$ and $\left.150 \mathrm{mg} \mathrm{L}^{-1}\right)$ of paclobutrazol (PBZ) on growth, side shoot emission and yield of two tomato hybrids (Jennifer and AF 7631), cultivated in open field and in protected environment. The work was conducted from April to October of 2007, at UNESP - Campus of Ilha Solteira, State of São Paulo. The PBZ was applied to seedlings 15 days after sowing, and its effects were evaluated, considering the tested concentrations, for tomato plants after the transplant. A randomized blocks design was used, in a factorial scheme, with analysis for series of experiments, in a fixed model, consisting of 4 replicates for environment and 9 plants per plot. As results, the hybrid Jennifer showed higher rates of absolute growth, resulting in higher plants than $\mathrm{AF} 7631$, on the $60^{\text {th }}$ day after the transplantation, but, with no differences between them regarding side shooting and crop yield. In the greenhouse, higher plants were obtained, with higher side shooting and higher yield than that observed in the open field. The use of increasing concentrations of paclobutrazol reduced the height of plants and their growth rate, decreased side shooting, and reduced the crop yield.

Index terms: Solanum lycopersicum L., growth regulator, greenhouse.

(Recebido em 24 de outubro de 2008 e aprovado em 18 de agosto de 2010)

\section{INTRODUÇÃO}

A cultura do tomateiro destaca-se, mundialmente, por sua importância econômica, social e por sua versatilidade de uso (Fontes \& Silva, 2005). No Brasil, é a segunda olerácea em importância econômica. Anualmente, são cultivados 56.678 ha, com uma produção estimada em 3.364.438 toneladas de frutos (FOOD AND AGRICULTURE ORGANIZATION OF THE UNITED NATIONS - FAO, 2009). Em 2006, o estado de São Paulo produziu cerca de 20,5 milhões de caixas de tomate com $25 \mathrm{~kg}$ de frutos em uma área de, aproximadamente, 8.555,86 ha (Instituto de Economia Agrícola, 2008).
Trabalhos de pesquisa (Gualberto et al., 2002) têm comprovado incrementos de rendimentos e qualidade no cultivo protegido, além da possibilidade de produção de entressafra, o que tem tornado essa técnica bastante difundida entre os agricultores.

Todavia demanda grande investimento na construção da estrutura física e desse modo, o que se espera é que as áreas cobertas sejam intensivamente utilizadas, com otimização da produção. Nesse aspecto, a utilização de reguladores de crescimento pode ser uma opção para obtenção de plantas mais compactas, possibilitando um arranjo espacial que resulte em maiores

${ }^{1}$ Parte da Tese de Doutorado do primeiro autor

2Universidade Estadual de Mato Grosso do Sul/UEMS - Unidade Universitária de Aquidauana - Aquidauana, MS

3Universidade Estadual Paulista Júlio de Mesquita Filho/UNESP - Departamento de Apoio, Produção e Saúde Animal/DAPSA - Rua Clóvis Pestana - 793 -

Dona Amélia - 16050-680 - Araçatuba, SP - maxfaria@fmva.unesp.br 
produtividades, além de reduzir o número de operações de desbrota.

Os reguladores de crescimento são utilizados na agricultura com o intuito de reduzir o crescimento vegetativo, promover os processos de floração e frutificação, além de regular a alternância da produção (Silva et al., 2003). Porém, o grau de resposta desses reguladores varia com a espécie, com a cultivar, com o método de aplicação e com a concentração utilizada. Entre esses compostos está o paclobutrazol, que em geral tem proporcionado aumento na produtividade.

Desde o seu descobrimento, o PBZ tem sido amplamente estudado na agricultura e seus efeitos tem sido relatado em inúmeras espécies vegetais, com diferentes padrões de crescimento, obtendo-se resultados que vão muito além da simples redução do alongamento do caule, em resposta à inibição da biossíntese de GA (ácido giberélico) (Seleguini, 2007).

O paclobutrazol (PBZ) bloqueia a biossíntese de GA diminuindo o desenvolvimento das plantas. Esses inibidores são capazes de induzir o florescimento sob condições não indutivas (Santos et al., 2004). Entretanto, alguns efeitos adversos como inibição do florescimento e redução da frutificação, também, são relatados na literatura, porém, esses efeitos variam em função de fatores como doses, o modo e o momento da aplicação, cultivar utilizada, estádio fenológico, condições climáticas, entre outros.

De acordo com Resende \& Souza (2002), o uso de reguladores de crescimento em cultivares de hortaliças tem se tornado uma opção para controlar, sobretudo, a altura de plantas, contudo, pouco se sabe sobre os efeitos do paclobutrazol nos processos fisiológicos das plantas que possam contribuir para o incremento da produtividade. Já Berova \& Zlatev (2000) constataram que o paclobutrazol reduz a altura da planta e aumenta a espessura do talo da planta jovem de tomate, como também acelera a formação de raiz, o que se torna uma vantagem, pois melhora a qualidade das mudas para o plantio.

Giovinazzo \& Souza-Machado (2001) utilizaram o paclobutrazol como tratamento em semente de tomate para o cultivo em estufa com a finalidade de testar o vigor, a tolerância ao stress, sob condições de temperatura baixa e precocidade de colheita, e constataram aumentos significativos do rendimento em $13 \%$ devido ao tratamento com paclobutrazol, juntamente com antecipação no início da colheita em $6 \%$.

Objetivou-se, neste estudo, avaliar o efeito de diferentes concentrações do regulador de crescimento paclobutrazol (PBZ) sobre o crescimento, a emissão de brotos laterais e a produtividade de dois híbridos de tomateiro de crescimento indeterminado, em campo aberto e em abrigo para cultivo protegido, em Ilha Solteira (SP).

\section{MATERIAL E MÉTODOS}

O trabalho foi desenvolvido na Fazenda de Ensino, Pesquisa e Extensão da UNESP, Campus de Ilha SolteiraSP ( $20^{\circ} 22^{\prime} \mathrm{S} ; 51^{\circ} 22^{\prime} \mathrm{W}$ e altitude de $\left.330 \mathrm{~m}\right)$. Avaliaram-se os efeitos da aplicação de paclobutrazol por aspersão, nas concentrações de 0; 50; 100 e $150 \mathrm{mg} \mathrm{L}^{-1}$, em mudas de tomateiro dos híbridos Jennifer e AF 7631, ambos do tipo salada, longa-vida e de crescimento indeterminado, em dois ensaios conduzidos, simultaneamente, em campo aberto e em ambiente protegido.

Cada ensaio foi estabelecido no delineamento em blocos casualizados, em esquema fatorial (concentrações de paclobutrazol $\mathrm{x}$ híbridos de tomateiro), com quatro blocos (linhas de cultivo) e nove plantas por parcela. Os resultados foram submetidos à análise de variância para grupo de experimentos (Banzatto \& Kronka, 2006), com o intuito de se determinar, também, os efeitos dos locais de cultivo (ambiente protegido e campo aberto).

O abrigo para cultivo protegido tinha orientação leste-oeste, dimensões de 5,4 x 30,0 m, pé direito de 2,4 m, cobertura com filme de polietileno transparente de $75 \mu \mathrm{m}$ de espessura e perímetro cercado com tela de sombreamento de $50 \%$, até $1,5 \mathrm{~m}$ de altura.

O solo da área experimental foi classificado como Argissolo Vermelho Eutrófico e as análises de fertilidade, de 0 a $20 \mathrm{~cm}$, apresentaram os seguintes resultados: a) condições de ambiente protegido: $324 \mathrm{mg} \mathrm{dm}^{-3}$ de $\mathrm{P}$ (resina); $31 \mathrm{~g} \mathrm{dm}^{-3} \mathrm{de}$ M.O.; 5,6 de $\mathrm{pH}\left(\mathrm{CaCl}_{2}\right) ; 2,1 \mathrm{mmol}_{\mathrm{c}} \mathrm{dm}^{-3}$ de $\mathrm{K} ; 84 \mathrm{mmol} \mathrm{dm}^{-3} \mathrm{de} \mathrm{Ca} ; 17 \mathrm{mmol}_{\mathrm{cm}}^{-3} \mathrm{de} \mathrm{Mg}$; $25 \mathrm{mmol} \mathrm{dm}^{-3} \mathrm{de} \mathrm{H}+\mathrm{Al} ; 127,7 \mathrm{mmol} \mathrm{dm}^{-3} \mathrm{de}$ CTC e $80 \% \mathrm{de}$ saturação por bases; b) condições de campo aberto: $42 \mathrm{mg} \mathrm{dm}^{-3}$ de P (resina); $20 \mathrm{~g} \mathrm{dm}^{-3}$ de M.O.; 5,4 de $\mathrm{pH}\left(\mathrm{CaCl}_{2}\right)$; $1,8 \mathrm{mmol}_{\mathrm{c}} \mathrm{dm}^{-3} \mathrm{de} \mathrm{K} ; 43 \mathrm{mmol}_{\mathrm{c}} \mathrm{dm}^{-3} \mathrm{de} \mathrm{Ca} ; 10 \mathrm{mmol}_{\mathrm{c}} \mathrm{dm}^{-3} \mathrm{de}$ $\mathrm{Mg} ; 22 \mathrm{mmol}_{\mathrm{c}} \mathrm{dm}^{-3} \mathrm{de} \mathrm{H}+\mathrm{Al} ; 76,6 \mathrm{mmol}_{\mathrm{c}} \mathrm{dm}^{-3} \mathrm{de}$ CTC e $71 \%$ de saturação por bases.

A semeadura ocorreu no dia 16/04/2007, em bandejas de poliestireno expandido com 200 células, utilizando substrato organo-mineral comercial. Aos 15 dias após a emergência, procedeu-se à aplicação do regulador de crescimento, através da aspersão de $5 \mathrm{~mL}$ de solução por planta, nas concentrações específicas de cada tratamento, com o auxílio de um pulverizador de pressão, com capacidade de 1,25 L. No momento da aplicação, a parcela tratada foi protegida para evitar a contaminação das demais por deriva.

As adubações de plantio, conforme recomendação de Raij et al. (1997), consistiram na aplicação de $14 \mathrm{~g} \mathrm{~m}^{-2} \mathrm{de}$ 
uréia e $35 \mathrm{~g} \mathrm{~m}^{-2}$ de $\mathrm{KCl}$, na área sob proteção plástica, enquanto em campo aberto, foram aplicados $78,8 \mathrm{~g} \mathrm{~m}^{-2} \mathrm{de}$ calcário calcinado em área total, e foram utilizados $250 \mathrm{~g} \mathrm{~m}^{-2}$ de fertilizante com fórmula 4-30-10 e $50 \mathrm{~g} \mathrm{~m}^{-2}$ de superfosfato simples.

Os canteiros foram levantados com $0,15 \mathrm{~m}$ de altura e 0,40 m de largura, instalando-se, em cada um deles, duas linhas de fitas gotejadoras com vazão nominal individual de $3,8 \mathrm{~L} \mathrm{~h}^{-1} \mathrm{~m}^{-1}$ (a $70 \mathrm{kPa}$ ), para irrigação das plantas.

As mudas foram transplantadas, em ambos os ambientes, no dia 28/05/07, no espaçamento de 1,0 x 0,4 m, e as plantas foram conduzidas com haste única, tutoradas em sistema de espaldeira simples, realizando-se a poda apical após a sétima inflorescência.

As adubações de cobertura, comuns aos ambientes de cultivo e realizadas em 25/06, 25/07, 15/08 e 06/09/07, consistiram na aplicação de $12 \mathrm{~g} \mathrm{~m}^{-2}$ de uréia e $8 \mathrm{~g} \mathrm{~m}^{-2}$ de $\mathrm{KCl}$, de acordo com a recomendação de adubação para o estado de São Paulo (Raij et al., 1997).

As plantas foram irrigadas em três turnos diários de 10 minutos até o início da frutificação e, a partir de então, em quatro turnos de 10 minutos. O tratamento fitossanitário foi o recomendado para a cultura, para o adequado controle de pragas e doenças. A colheita foi realizada duas vezes por semana, no período de 31/07 a 01/10/2007.

As condições microclimáticas foram monitoradas e as médias dos totais diários de radiação solar global foram de 11,4 $\mathrm{MJ} \mathrm{m}^{-2} \mathrm{dia}^{-1}$ e 18,6 $\mathrm{MJ} \mathrm{m}^{-2} \mathrm{dia}^{-1}$; as temperaturas médias do ar foram de $22,9^{\circ} \mathrm{C}$ e $23,4^{\circ} \mathrm{C}$ e as umidades relativas médias do ar foram de $58 \%$ e $54 \%$; respectivamente, no ambiente protegido e em campo aberto, para o período de 28/05 a 01/10/2007. O total de precipitação pluviométrica, no mesmo período, foi de $51,8 \mathrm{~mm}$.

Foram avaliados a altura média de plantas aos 60 dias após o transplantio, a taxa média de crescimento absoluto, o número médio de brotos laterais por planta, a massa média de matéria seca da brotação lateral por planta, o número médio de frutos por unidade de área, a massa média de matéria fresca do fruto, a produtividade média e a distribuição porcentual da produção, de acordo com a classificação dos frutos por classe de tamanho, em função do diâmetro transversal $(\varnothing)$, em frutos pequenos $(50 \leq \varnothing<65 \mathrm{~mm})$, médios $(65 \leq \varnothing<80 \mathrm{~mm})$, grandes $(80 \leq \varnothing<100 \mathrm{~mm})$ e gigantes $(\varnothing \geq 100 \mathrm{~mm})$.

Os resultados foram submetidos à análise de variância, comparando-se as médias de híbridos e de ambientes pelo teste de Tukey $(\mathrm{P}<0,05)$, e realizando-se as análises de regressão para descrição dos efeitos das concentrações de paclobutrazol.

\section{RESULTADOS E DISCUSSÃO}

Aos 60 dias após o transplantio, independente do ambiente de cultivo e da concentração de paclobutrazol (PBZ) empregada, o híbrido Jennifer apresentou altura média de plantas significativamente maior que aquela do híbrido AF 7631, consequência de uma taxa de crescimento absoluto, em média, cerca de $8 \%$ superior (Tabela 1).

Ainda, em relação à altura de plantas, houve interação entre o ambiente de cultivo e a concentração do regulador de crescimento utilizada. Foram obtidas plantas significativamente mais altas no cultivo protegido, para todas as concentrações de PBZ testadas, o que foi reflexo das maiores taxas médias de crescimento absoluto observadas nesse ambiente (Tabela 2). Esse resultado está relacionado às alterações ambientais proporcionadas pelo uso de filme plástico, principalmente no que se refere à radiação, tanto em termos quantitativos, por determinar redução em sua disponibilidade em relação ao campo aberto (na média, de 18,6 para 11,4 $\mathrm{MJ} \mathrm{m}^{-2} \mathrm{dia}^{-1}$, como medido neste ensaio, correspondendo a um sombreamento de 38,7\%), como qualitativos, pela modificação no espectro radiação que alcança as plantas, com alteração no balanço dos comprimentos de onda na faixa do vermelho $(660 \mathrm{~nm})$ e do vermelho-distante $(730 \mathrm{~nm})$, que atuam sobre o equilíbrio entre as formas ativas e inativas dos fitocromos, com efeito sobre a morfologia das plantas (Kittas \& Baille, 1998; Kittas et al., 1999; Reisser Junior et al., 2003).

O uso de paclobutrazol foi efetivo ao reduzir o porte das plantas de tomateiro. Em campo aberto, o aumento na concentração de PBZ promoveu reduções lineares na taxa de crescimento absoluto e na altura das plantas, aos 60 dias após o transplantio, enquanto em ambiente protegido, em ambos os casos, as respostas se ajustaram a uma regressão de segundo grau (Tabela 2 e Figura 1), com valores mínimos para concentrações em torno dos $150 \mathrm{mg} \mathrm{L}^{-1}$. O PBZ inibe a conversão de caureno para ácido caurenóico, reduzindo os níveis de todas as formas de ácidos giberélicos, assim, diminuindo a taxa de elongação e divisão celular (Almeida \& Pereira, 1997; Fletcher et al., 2000).

Constatou-se que os híbridos estudados não diferiram entre si quanto ao número de brotos laterais emitidos por planta e quanto à massa de matéria seca da brotação lateral (Tabela 1). Por outro lado, as plantas sob cultivo protegido apresentaram brotos significativamente mais vigorosos, com maior acúmulo de matéria seca do que aqueles de plantas cultivadas em campo aberto, embora sem diferir no número de brotos por planta (Tabela 1).

$\mathrm{O}$ incremento na concentração de PBZ, por sua vez, reduziu significativamente o número de brotos e a massa de matéria seca da brotação lateral (Tabela 1), segundo 
ajustes polinomiais quadráticos, apresentados na Figura 1. O número mínimo de brotos e a menor massa de matéria seca da brotação lateral foram estimados, respectivamente, para concentrações de 145 e $113 \mathrm{mg} \mathrm{L}^{-1}$ de paclobutrazol.

A utilização do regulador de crescimento determinou decréscimos, em média, de 36,48 e $56 \%$ no número de brotos laterais, e de 52, 55 e $58 \%$ na massa de matéria seca da brotação lateral, respectivamente, nas concentrações de 50, 100 e $150 \mathrm{mg} \mathrm{L}^{-1}$, resultados superiores aos obtidos por Seleguini (2007), ao avaliar o efeito do PBZ no híbrido de tomateiro AF 7631, nas concentrações de 50 e $100 \mathrm{mg} \mathrm{L}^{-1}$.

Os híbridos não diferiram no número de frutos produzidos por unidade de área, porém o híbrido Jennifer produziu frutos com maior massa média, sem que isso fosse suficiente para determinar diferenças significativas de produtividade entre os materiais genéticos estudados, como indicado na Tabela 3.

Tabela 1 - Altura média de plantas (AP), aos 60 dias após o transplantio, taxa média de crescimento absoluto (TCA), número médio de brotos por planta (NBP) e massa média de matéria seca da brotação lateral (MSBL), obtidos para os híbridos de tomateiro AF 7631 e Jennifer, tratados com diferentes concentrações de paclobutrazol (PBZ), em cultivo protegido e em campo aberto. Ilha Solteira (SP), 2007.

\begin{tabular}{|c|c|c|c|c|}
\hline FATOR & $\begin{array}{l}\text { AP } \\
(\mathrm{cm})\end{array}$ & $\begin{array}{c}\text { TCA } \\
\left(\mathrm{cm} \mathrm{dia}^{-1}\right)\end{array}$ & NBP & $\begin{array}{c}\text { MSBL } \\
\left(\mathrm{g} \mathrm{planta}^{-1}\right)\end{array}$ \\
\hline \multicolumn{5}{|l|}{ Híbridos } \\
\hline AF 7631 & $106,72 \mathrm{a}$ & $1,63 \mathrm{a}$ & $9,10 \mathrm{a}$ & $19,77 \mathrm{a}$ \\
\hline Jennifer & $114,26 \mathrm{~b}$ & $1,76 \mathrm{~b}$ & $10,11 \mathrm{a}$ & $21,35 \mathrm{a}$ \\
\hline \multicolumn{5}{|l|}{ Ambiente } \\
\hline Protegido & (1) & (1) & $10,06 \mathrm{a}$ & $21,61 \mathrm{a}$ \\
\hline Campo & -- & -- & $9,07 \mathrm{a}$ & $19,52 \mathrm{~b}$ \\
\hline \multicolumn{5}{|l|}{$\operatorname{PBZ}\left(\mathrm{mg} \mathrm{L}^{-1}\right)$} \\
\hline 0 & (1) & (1) & $14,72^{\mathrm{RQ}}$ & $35,21^{\mathrm{RQ}}$ \\
\hline 50 & -- & -- & 9,38 & 16,72 \\
\hline 100 & -- & -- & 7,64 & 15,80 \\
\hline 150 & -- & -- & 6,50 & 14,51 \\
\hline $\mathrm{CV}(\%)$ & 12,39 & 13,47 & 25,89 & 18,17 \\
\hline
\end{tabular}

Médias seguidas pelas mesmas letras, nas colunas, dentro de cada fator, não diferem entre si pelo teste de Tukey $(\mathrm{P}>0,05)$.

(1) Interação significativa entre o fator ambiente e o fator concentração de PBZ.

RL - regressão linear significativa; RQ - regressão quadrática significativa.

Tabela 2 - Altura média de plantas (AP), aos 60 dias após o transplantio, e taxa média de crescimento absoluto (TCA), obtidos para os híbridos de tomateiro AF 7631 e Jennifer, tratados com diferentes concentrações de paclobutrazol (PBZ), em cultivo protegido e em campo aberto. Ilha Solteira (SP), 2007.

\begin{tabular}{ccccc}
\hline \multirow{2}{*}{ PBZ } & \multicolumn{2}{c}{$\begin{array}{c}\text { AP } \\
(\mathrm{cm})\end{array}$} & \multicolumn{2}{c}{$\begin{array}{c}\text { TCA } \\
\left(\mathrm{cm} \mathrm{dia}^{-1}\right)\end{array}$} \\
\cline { 2 - 5 }$\left(\mathrm{mg} \mathrm{L}^{-1}\right)$ & Ambiente protegido & Campo & Ambiente protegido & Campo \\
\hline 0 & $165,55 \mathrm{a}$ & $114,0 \mathrm{~b}$ & $2,56 \mathrm{a}$ & $1,71 \mathrm{~b}$ \\
50 & $118,65 \mathrm{a}$ & $97,71 \mathrm{~b}$ & $1,84 \mathrm{a}$ & $1,50 \mathrm{~b}$ \\
100 & $119,35 \mathrm{a}$ & $89,33 \mathrm{~b}$ & $1,86 \mathrm{a}$ & $1,36 \mathrm{~b}$ \\
150 & $98,60 \mathrm{a}$ & $80,73 \mathrm{~b}$ & $1,52 \mathrm{a}$ & $1,22 \mathrm{~b}$ \\
\hline Regressões & RQ & RL & RQ & RL \\
\hline
\end{tabular}

Médias seguidas pelas mesmas letras, nas linhas, em cada característica estudada, não diferem entre si pelo teste de Tukey (P>0,05). $\mathrm{RL}$ - regressão linear significativa; $\mathrm{RQ}$ - regressão quadrática significativa. 

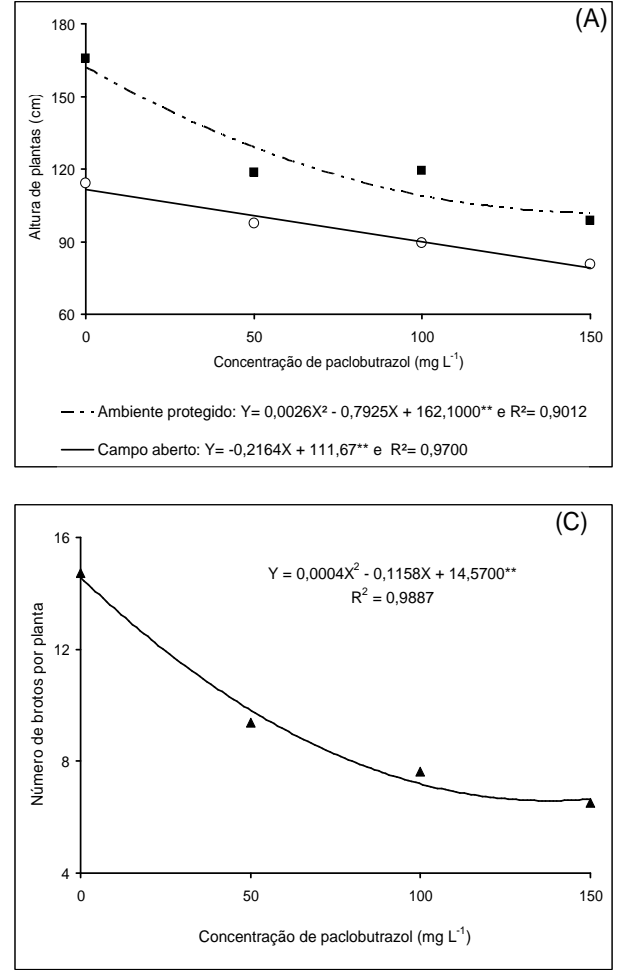

(C)

* - significativo $(\mathrm{P}<0,05)$ e ** - significativo $(\mathrm{P}<0,01)$.

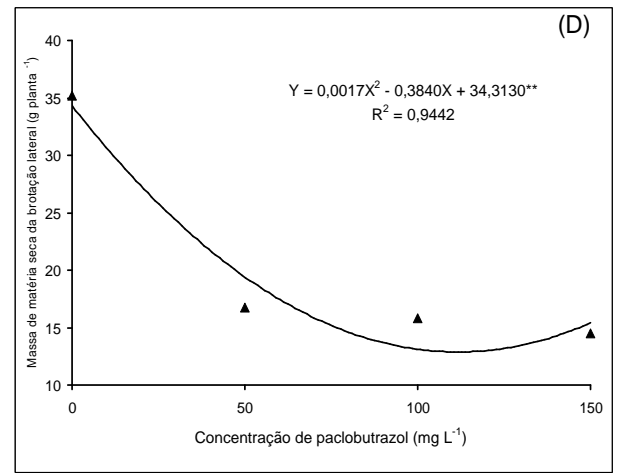

(B)

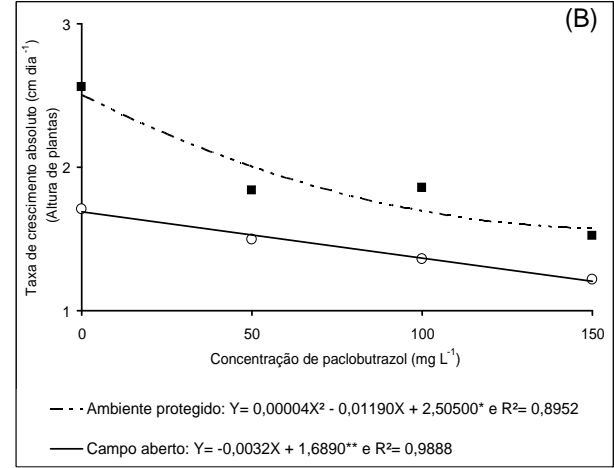

(D)

Figura 1 - Variação da altura de plantas aos 60 dias após o transplantio (A) e de sua taxa de crescimento absoluto (B), do número de brotos por planta (C) e da massa de matéria seca da brotação lateral (D), em função da concentração de paclobutrazol, para os híbridos de tomateiro Jennifer e AF 7631, cultivados em campo aberto e em ambiente protegido, em Ilha Solteira (SP), em 2007.

Tabela 3 - Número médio de frutos por área, massa média de matéria fresca de fruto e produtividade média obtidos para os híbridos de tomateiro AF 7631 e Jennifer, tratados com diferentes concentrações de paclobutrazol (PBZ), em cultivo protegido e em campo aberto. Ilha Solteira (SP), 2007.

\begin{tabular}{lccc}
\hline FATOR & $\begin{array}{c}\text { Número médio de frutos } \\
\text { por } \mathrm{m}^{2}\end{array}$ & $\begin{array}{c}\text { Massa média de matéria } \\
\text { fresca de fruto }(\mathrm{g})\end{array}$ & $\begin{array}{c}\text { Produtividade média } \\
\left(\mathrm{kg} \mathrm{m}^{-2}\right)\end{array}$ \\
\hline Híbridos & & & $10,53 \mathrm{a}$ \\
AF 7631 & $64,25 \mathrm{a}$ & $162,33 \mathrm{~b}$ & $10,34 \mathrm{a}$ \\
Jennifer & $60,77 \mathrm{a}$ & $169,26 \mathrm{a}$ & $10,96 \mathrm{a}$ \\
\hline Ambiente & & & $9,90 \mathrm{~b}$ \\
Protegido & $63,92 \mathrm{a}$ & $170,57 \mathrm{a}$ & $12,40^{\mathrm{RQ}}$ \\
Campo & $61,10 \mathrm{a}$ & $161,02 \mathrm{~b}$ & 9,56 \\
\hline PBZ $\left(\mathrm{mg} \mathrm{L}^{-1}\right)$ & & & 10,38 \\
0 & $70,05^{\mathrm{RL}}$ & $176,17^{\mathrm{RL}}$ & 9,39 \\
50 & 59,06 & 160,96 & 16,67 \\
100 & 62,03 & 166,04 & 160,01 \\
\hline CV $(\%)$ & 58,91 & 7,16 & 16 \\
\hline
\end{tabular}

Médias seguidas pelas mesmas letras, nas colunas, dentro de cada fator, não diferem entre si pelo teste de Tukey $(\mathrm{P}>0,05)$. $\mathrm{RL}$ - regressão linear significativa; RQ - regressão quadrática significativa. 
O ambiente de cultivo não influenciou o número de frutos produzidos por unidade de área, todavia o cultivo protegido possibilitou a obtenção de frutos com maior massa média, o que resultou em produtividade significativamente superior àquela verificada em campo aberto (Tabela 3). Esses resultados são convergentes com os de Gualberto et al. (2002), que, também, observaram maiores rendimentos da cultura do tomateiro em ambiente protegido, quando comparado à produção a céu aberto.

Foi constatado que o número de frutos por unidade de área e a massa média do fruto decresceram linearmente com o incremento na concentração de PBZ empregada (Tabela 3 e Figura 2), impactando de forma negativa a produção da cultura. Assim, houve redução da produtividade, conforme um ajuste polinomial de segunda ordem, até um valor mínimo estimado para a concentração de $110 \mathrm{mg} \mathrm{L}^{-1}$ (Tabela $3 \mathrm{e}$ Figura 2), resultados semelhantes aos de Berova \& Zlatev (2000), que observaram que a aplicação foliar de PBZ reduziu a produtividade em 9,6\%, porém divergentes daqueles de Souza-Machado et al. (1999) e Seleguini (2007), que não constataram alterações no rendimento do tomateiro com a aplicação do mesmo regulador de crescimento.

Constatou-se que o híbrido Jennifer teve produção superior de frutos médios e grandes, de maior valor comercial, e menor produção de frutos pequenos que o híbrido AF 7631, embora, em ambos os casos, a maior parcela da produção (ao redor de $90 \%$ ) tenha correspondido àquela de frutos pequenos e médios (Tabela 4). Os híbridos testados exibiram produção desprezível de frutos classificados como gigantes, não apresentando diferença entre si (Tabela 4).

O cultivo protegido possibilitou a obtenção de maiores produções de frutos classificados como médios e grandes, reduzindo significativamente a produção de frutos pequenos, em relação ao cultivo em campo aberto, sem nenhum efeito, todavia, sobre a produção de frutos gigantes (Tabela 4).
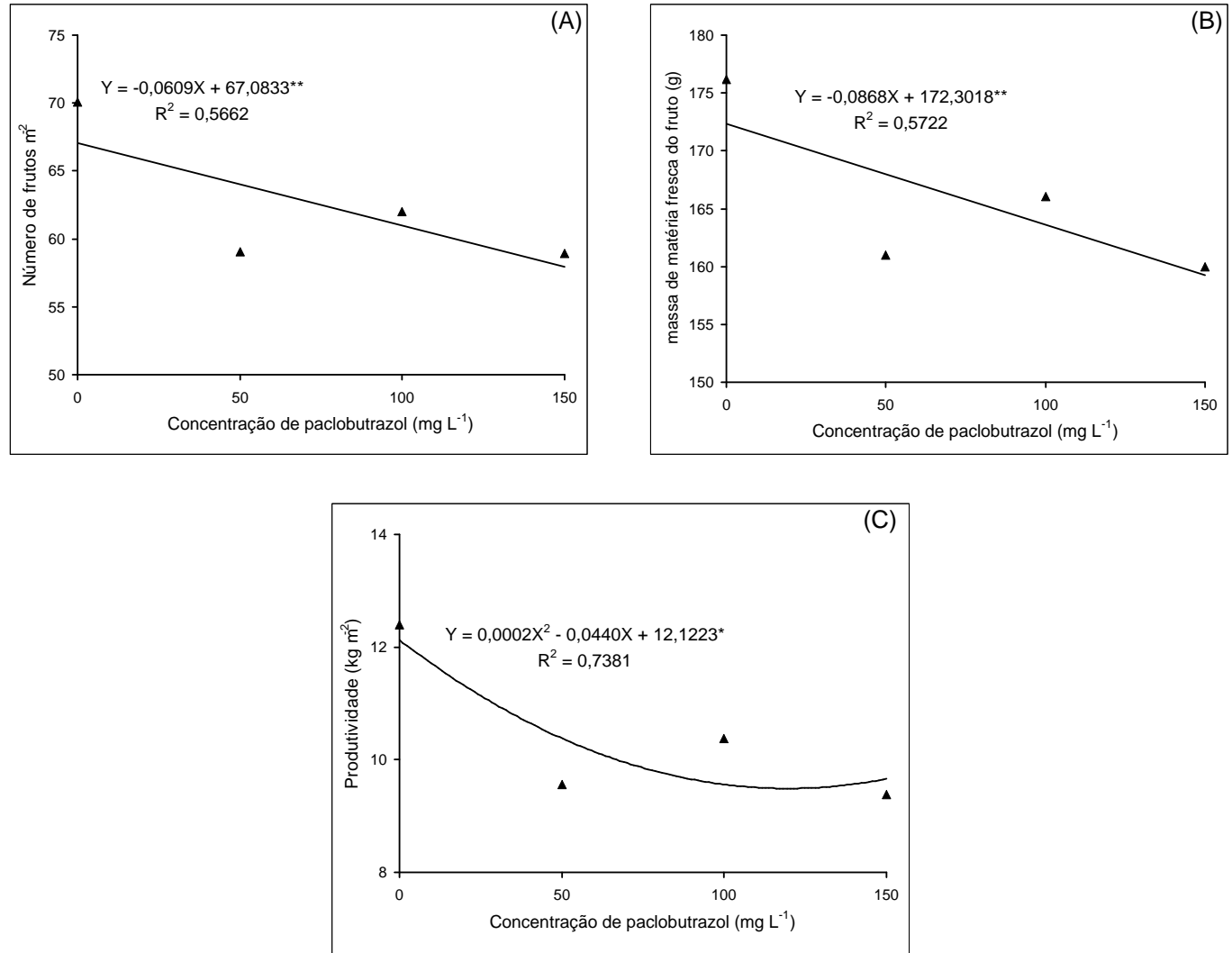

* - significativo $(\mathrm{P}<0,05)$ e ** - significativo $(\mathrm{P}<0,01)$.

Figura 2 - Variação do número médio de frutos por unidade de área (A), da massa média de matéria fresca do fruto (B) e da produtividade média da cultura, em função da concentração de paclobutrazol, para os híbridos de tomateiro Jennifer e AF 7631, cultivados em campo aberto e em ambiente protegido. Ilha Solteira (SP), 2007. 
Tabela 4 - Dados transformados da distribuição porcentual da produção de acordo com a classificação dos frutos por tamanho, obtidas para os híbridos de tomate AF 7631 e Jennifer, tratados com diferentes concentrações de paclobutrazol (PBZ), em cultivo protegido e em campo aberto. Ilha Solteira (SP), 2007.

\begin{tabular}{|c|c|c|c|c|}
\hline \multirow{2}{*}{ FATOR } & \multicolumn{4}{|c|}{ Classificação dos frutos por tamanho ${ }^{(1)}$} \\
\hline & Pequenos ${ }^{(2)}$ & Médios ${ }^{(2)}$ & Grandes $^{(2)}$ & Gigantes $^{(2)}$ \\
\hline \multicolumn{5}{|l|}{ Híbridos } \\
\hline AF 7631 & 46,15 a $(51,5 \%)$ & $40,39 b(41,6 \%)$ & $14,95 \mathrm{~b} \quad(6,9 \%)$ & 4,20 a $(0,0)$ \\
\hline Jennifer & 41,32 b $(43,2 \%)$ & 43,83 a $(47,5 \%)$ & 17,11 a $(9,3 \%)$ & 4,11 a $(0,0)$ \\
\hline \multicolumn{5}{|l|}{ Ambiente } \\
\hline Estufa & 41,14 b $(42,9 \%)$ & 43,60 a $(47,1 \%)$ & 18,29 a $(10,0 \%)$ & 4,15 a $(0,0)$ \\
\hline Campo & 46,33 a $(51,8 \%)$ & $40,63 \mathrm{~b}(42,0 \%)$ & $13,77 \mathrm{~b} \quad(6,2 \%)$ & 4,15 a $(0,0)$ \\
\hline \multicolumn{5}{|l|}{$\operatorname{PBZ}\left(\mathrm{mg} \mathrm{L}^{-1}\right)$} \\
\hline 0 & $38,85^{(3)}(39,0 \%)$ & $44,06(47,9 \%)$ & $21,25^{(4)}(13,0 \%)$ & $4,24(0,1 \%)$ \\
\hline 50 & $46,62 \quad(52,3 \%)$ & $40,29(41,4 \%)$ & $(6,3 \%)$ & $4,16(0,0)$ \\
\hline 100 & $42,97 \quad(46,0 \%)$ & $43,13(46,3 \%)$ & $(7,7 \%)$ & $4,05(0,0)$ \\
\hline 150 & $46,50 \quad(52,1 \%)$ & $40,97(42,6 \%)$ & $(5,3 \%)$ & $4,15(0,0)$ \\
\hline C.V. $(\%)$ & 12,14 & 9,63 & 27,64 & 11,25 \\
\hline
\end{tabular}

Médias seguidas pelas mesmas letras, nas colunas, dentro de cada fator, não diferem entre si pelo teste de Tukey $(\mathrm{P}>0,05)$. ${ }^{(1)}$ Dados transformados em $\operatorname{arcsen} \sqrt{(x+0,5) / 100}$.

${ }^{(2)}$ Pequenos: $50 \leq \varnothing<65 \mathrm{~mm}$; médios: $65 \leq \varnothing<80 \mathrm{~mm}$; grandes: $80 \leq \varnothing<100 \mathrm{~mm}$; gigantes: $\geq 100 \mathrm{~mm}$; em que $\varnothing$ é o diâmetro transversal do fruto.

${ }^{(3)} \mathrm{Y}_{1}=0,0386 \mathrm{X}+40,843 * *$ e $\mathrm{R}^{2}=0,4599(* *$ - significativa, com $\mathrm{P}<0,01)$.

${ }^{(4)} \mathrm{Y}_{2}=0,0005 \mathrm{X}^{2}-0,1195 \mathrm{X}+20,6260 * \mathrm{e} \mathrm{R}^{2}=0,7998$ (*- significativa, com $\left.\mathrm{P}<0,05\right)$.

Valores em percentagem, entre parênteses, correspondem às médias originais, apenas para ilustração.

Em que pese o seu efeito positivo sobre a produção de frutos com melhor classificação comercial para consumo in natura, ainda assim, a maior parcela da produção, em torno de $90 \%$, foi de frutos pequenos e médios, sem grande destaque em relação ao que foi obtido a céu aberto.

Como efeito do regulador do crescimento, observouse que o incremento em sua concentração determinou um aumento linear do porcentual de frutos pequenos e uma redução no porcentual de frutos grandes, segundo um ajuste polinomial quadrático (Tabela 4), com valor mínimo para a concentração de $120 \mathrm{mg} \mathrm{L}^{-1}$ de PBZ, resultando, portanto, em prejuízo de caráter qualitativo da produção, como, também, observado por Seleguini (2007), além daquele quantitativo, referente à redução de produtividade.

\section{CONCLUSÕES}

O paclobutrazol reduziu a altura e a brotação lateral de plantas de tomateiro, em intensidades que variaram com as concentrações testadas, porém, a produção de tomates, tanto em termos quantitativos como qualitativos (tamanho dos frutos), foi afetada negativamente pelo uso do regulador de crescimento, também, em níveis dependentes de sua concentração.
Os híbridos de tomateiro estudados apresentaram produtividades equivalentes, todavia, o híbrido Jennifer produziu frutos de maior massa média, além de maior porcentual de frutos classificados como grandes e médios, em relação ao híbrido AF 7631.

O cultivo protegido resultou em plantas mais vigorosas, com maior produtividade e porcentuais de frutos grandes e médios mais elevados, em comparação ao cultivo em campo aberto.

Frente aos resultados, com o uso de paclobutrazol, abrem-se possibilidades para redução das operações de desbrota e para o adensamento de plantas.

\section{AGRADECIMENTOS}

À Capes, pela concessão de bolsa de doutorado, e à Sakata Seed Sudamerica, pela doação das sementes.

\section{REFERÊNCIAS BIBLIOGRÁFICAS}

ALMEIDA, J.A.S.; PEREIRA, M.F.D.A. Efeito de $\mathrm{GA}_{3} \mathrm{e}$ paclobutrazol no desenvolvimento vegetativo do girassol. Revista Brasileira de Fisiologia Vegetal, Brasília, v.9, n.1, p.55-60, 1997. 
BANZATTO, D.A.; KRONKA, S.N. Experimentação agrícola. 4.ed. Jaboticabal: FUNEP, 2006. 237p.

BEROVA, M.; ZLATEV, Z. Physiological response and yield of paclobutrazol treated tomato plants (Lycopersicon esculentum Mill.). Plant Growth Regulation, Dordrecht, v.30, p.117-123, 2000.

FLETCHER, R.A.; GILLEY, A.; SANKHLA, N.; DAVIS, T.D. Triazoles as plant growth regulators and stress protectants. Horticultural Reviews, New York, v.24, p.55-138, 2000.

FONTES, P.C.R.; SILVA, D.J.H. Cultura do tomate. In: REZENDE, P.C. Olericultura: teoria e prática. Viçosa, MG: UFV, 2005. p.457-475.

FOOD AND AGRICULTURE ORGANIZATION OFTHE UNITED NATIONS. Disponível em: ⿶http://faostat.faó. org/site/567/DesktopDefault.aspx?PageID=567\#ancor>. Acesso em: 11 ago. 2010.

GIOVINAZZO, R.; SOUZA-MACHADO, V. Paclobutrazol responses with processing tomato in France. Acta Horticulturae, Wageningen, v.542, p.355-358, 2001.

GUALBERTO, R.; BRAZ, L.T.; BANZATTO, D.A. Produtividade, adaptabilidade e estabilidade fenotípica de cultivares de tomateiro sob diferentes condições de ambiente. Pesquisa Agropecuária Brasileira, Brasília, v.37, n.1, p.81-88, 2002.

INSTITUTO DE ECONOMIA AGRÍCOLA. Área e produção dos principais produtos da agropecuária do Estado de São Paulo. Disponível em: <http:// IWww.iea.sp.gov.br/out/banco/menu.php⿳亠丷厂甲. Acesso em: 11 mar. 2008.

KITTAS, C.; BAILLE, A. Determination of the spectral properties of several greenhouse cover materials and evaluation of specific parameters related to plant response. Journal of Agricultural Engineering Research, London, v.71, n.2, p.193-202, 1998.

KITTAS, C.; BAILLE, A.; GIAGLARAS, P. Influence of covering material and shading on the spectral distribution of light in greenhouses. Journal of Agricultural Engineering Research, London, v.73, n.4, p.341-351, 1999.

RAIJ, B.V.; CANTARELLA, H.; QUAGGIO, J.A.; FURLANI, A.M.C. Recomendação de adubação e calagem para o Estado de São Paulo. Campinas: IAC, 1997. 285p. (Boletim técnico, 100).

REISSER JUNIOR, C.; BERGAMASCHI, H.; RADIN, B.; BERGONCI, J.I. Alterações morfológicas do tomateiro em resposta à redução de radiação solar em ambiente protegido. Revista Brasileira de Agrometeorologia, Santa Maria, v.11, n.1, p.7-14, 2004

RESENDE, G.M.; SOUZA, R.J. Efeito de doses de Paclobutrazol na cultura do alho. Pesquisa Agropecuária Brasileira, Brasília, v.37, n.5, p.637-641, 2002.

SANTOS, C.H.; KLAR, A.E.; GRASSI FILHO, H.; RODRIGUES, J.D.; PIERRE, F.C. Indução do florescimento e crescimento de tangerineira poncã (Citrus reticulata Blanco) em função da irrigação e da aplicação de paclobutrazol. Revista Brasileira de Fruticultura, Jaboticabal, v.26, n.1, p.8-12, 2004.

SELEGUINI, A. Uso de paclobutrazol na produção de mudas, no crescimento produção e qualidade de frutos de tomateiro em ambiente protegido. 2007. 100f. Tese (Doutorado em Agronomia, Sistema de Produção)-Universidade Estadual Paulista, Ilha Solteira, 2007.

SILVA, C.M.M.S.; FAY, E.F.; JONSSON, C.M.

Paclobutrazol: regulador de crescimento vegetal. In: SILVA, C.M.M.S.; FAY, E.F. (Eds.). Impacto ambiental do regulador de crescimento vegetal paclobutrazol. Jaguariúna: Embrapa-CNPMA, 2003. p.11-16. (Documentos, 30).

SOUZA-MACHADO, V.; PITBLADO, R.; ALI, A.; MAY, P. Paclobutrazol in tomato (Lycopersicon esculentum) for improved tolerance to early transplanting and earlier harvest maturity. Acta Horticulturae, Wageningen, v.487, p.139-143, 1999. 